\title{
Evaluation of the Turkish Highway Network Analysis with Traffic Data
}

\author{
İ. Türker
}

\begin{abstract}
As a complex geospatial structure, Turkish national highway transportation network is studied by the means of network science. We used the dataset retrieved from the KGM (Karayolları Genel Müdürlüğ̈̈) maps with a hand-driven process. The dataset labels the junctions in the map as nodes, and the roads between these junctions as edges. We outlined the statistical properties of the Turkish highway transportation network by the means of eigenvector, betweenness, closeness centrality, modularity and eccentricity measures, while comparative percentile plots between these measures are also performed. We investigated the correlation of these parameters with the traffic volume, and outlined that only eccentricity measure is correlated with the traffic volume. We also investigated the degree correlations of the network and found that the network displays disassortative mixing behavior, meaning that nodes with high degrees tend to connect with lower degree nodes, and vice versa. This property is consistent with the recent studies of transportation networks, as well as various types of real networks like Internet, World-Wide Web, protein interactions, neural network etc.
\end{abstract}

Index Terms - Transportation networks, Complex networks, Centrality, Data analysis, Scale-free networks.

\section{INTRODUCTION}

$\mathrm{N}^{\mathrm{s}}$ ETWORK science provides a framework to analyze the infrastructures of the networked systems in nature. These systems are in a variety spanning cellular [1,2], ecological [3, 4], social [5-8], WWW [9], power-grid networks [10, 11] etc. which exist as interconnected systems in nature. The characterization of such complex systems using statistical and computational techniques have attracted considerable attention in the literature in recent years. Complex systems are composed of numerous components, those interact in a manner that collective behavior is not an ordinal function of their individual behaviors [12].

As a complex system, transportation networks also deal with the nature of the movement patterns of people in a geographic region, where the movement is towards roads, railways or airways [13]. The studies in this field are performed in regional, national or global scale.

I. TÜRKER, Department of Computer Engineering; Karabük University, Karabük, Turkey, (e-mail: iturker@karabuk.edu.tr)

Manuscript received August 26, 2017; accepted December 28, 2017. DOI: $10.17694 /$ bajece.369235
Guimera et al. analyzed the structure of the worldwide air transportation network, a large-scale structure with a considerable impact on local, national, and international economies. They outlined that the most connected cities of the network are not necessarily the most central, resulting in anomalous values of the centrality [14]. In another study concerning air transportation networks, Bagler evaluated domestic civil aviation infrastructure of India as a complex network. He labeled the structure as a small-world network with scale-free property, as fingerprints of self-organizing phenomenon also reported in other transportation network studies. The mentioned network also displays disassortative mixing property, meaning that high degree nodes tend to connect with low degree nodes in majority [15].

$\mathrm{Xu}$ and Harriss stated out that the air transportation network of U.S. is also a small-world and scale-free network. They also outlined the emergence of a rich-club phenomenon which means cities with numerous connections tend to connect to cities with comparable degrees, which in turn sustains high volumes of traffic between these high degree nodes [16]. Also dissortative mixing patterns are observed by $\mathrm{Xu}$ and Harris, as reported in the study of Bagler.

Road and railway networks are other forms of transportation networks in which connections between nodes are affected by their spatial structures. In such networks connections between distant nodes can only be sustained via other nodes locating spatially between these distant nodes. This in turn results connections only established between spatially close locations. The studies in this aspect span several public transportation networks of regions like Singapore [17], Shanghai [18], Greece [19], Boston (U.S.) [20] etc. In the study of Soh et al., while two networks studied yield nearly neutral assortativity behaviors, bus network of Singapore is slightly disassortative while the fast railway network is slightly assortative [17].

Several network studies in transportation networks are also performed to investigate the impact of network structure in tourism [21], robustness in highway networks [22], capacity reliability road networks [23], urban street structures [24, 25] etc. The commonality of the mentioned studies are the evidence of universal properties of complex networks like being smallworld and scale-free, together with clustering coupled with a disassortative mixing property.

This study mainly focuses on uncovering the centrality and assortativity properties of the Turkish highway transportation network. The specifications of the dataset together with the results are detailed in the coming section, followed by a conclusion section. 


\section{DATA AND METHODS}

We can briefly abstract the basic network properties from that study as follows. The Turkish highway transportation network displays invincibly high node separation $(\sim 28.3)$ together with high diameter (83) values compared to the most real network structures. These high values are correlated with the spatial structure of the constructed network, in which the nodes are assumed as the junction points of the roads and the edges are the roads connecting these nodes. The network is also reported as having very high modularity $(0.918)$ together with low average clustering coefficient $(0.034)$. Further conclusions about the basic network analysis can be accessed via the mentioned study.

The network approach has a long tradition in economic geography and city planning, being used to investigate the territorial relationships among communication flows, population, wealth and land-uses [28]. Centrality is also a fundamental concept in network analysis, described as below:

i) Closeness centrality, $C^{C}$, measures how a node $i$ is close to the all other nodes along the shortest paths. It is defined as:

$$
C_{i}^{C}=\frac{N-1}{\sum_{j \in G ; j \neq i} d_{i j}}
$$

where $\mathrm{N}$ is the number of nodes and $d_{i j}$ is the shortest path length between nodes $i$ and $j$ in a network [28, 29].

ii) Betweenness Centrality, $C^{B}$ assumes a node is central if it is located between many other nodes, regarding the number of occurrences for that node as a hop between the shortest paths connecting the other nodes. The betweenness centrality of node $i$ is defined as:

$C_{i}^{B}=\frac{1}{(N-1)(N-2)} \sum_{j, k \in G, j \neq k \neq i} n_{j k}(i) / n_{j k}$

where $n_{j k}$ is the number of shortest paths between nodes $\mathrm{j}$ and $\mathrm{k}$, and $n_{j k}(i)$ is the number of shortest paths between nodes $j$ and $k$ which contain node $i[28,29]$.

iii) Eigenvector Centrality, $C^{E}$ defines the importance of a node as its connectivity to important nodes. It can also be assumed as a weighted sum of not only direct connections but indirect connections of every length. Thus, it takes into account the entire connectivity pattern in the network. Let A be the adjacency matrix for this graph; $a_{i j}=1$ if vertices $i$ and $j$ are connected by an edge and 0 if not.

$A x=\lambda x, \quad \lambda x_{i}=\sum_{j=1}^{n} a_{i j} x_{j}, \quad i=1, \ldots, n$

Eq. (3) describes eigenvector centrality $\mathrm{x}$ in two equivalent ways, as a matrix equation and as a sum. The centrality of a vertex is proportional to the sum of the centralities of the vertices to which it is connected. $\lambda$ is the largest eigenvalue of $\mathrm{A}$ and $\mathrm{n}$ is the number of vertices. [30].

Eccentricity $(E)$, a measure involving the distances between nodes is the maximum distance of a node to all other nodes in a network. Thus the minimum eccentricity of a network defines its radius whereas the maximum eccentricity defines its diameter [31]. The exact definition and formulation can be accessed via this Ref. This measure deviates from the average path length for a node by always taking into consideration the maximal shortest distances to the other nodes.

Another measure investigated in the network is Modularity $(M)$, quantifying the idea that true community structure in a network corresponds to a statistically surprising arrangement of edges. Modularity is the number of edges falling within groups minus the expected number in an equivalent network with edges placed at random. The precise mathematic formulation can be accessed at Ref. [32].

\section{RESULTS}

\subsection{Centrality, Modularity and Eccentricity}

To shed insight into the infrastructure of the network by the means of centrality, we calculated the centrality measures for the nodes by using the Gephi software introduced by Bastian [33]. The results are further analyzed in MATLAB to assign the number of nodes belonging to some pairs of Betweenness Centrality, Closeness Centrality, Eigenvector Centrality and Modularity Class measures, as percentiles of their maximal values. We present 3D percentile plots pairing these measures in Figures 1 to 3.

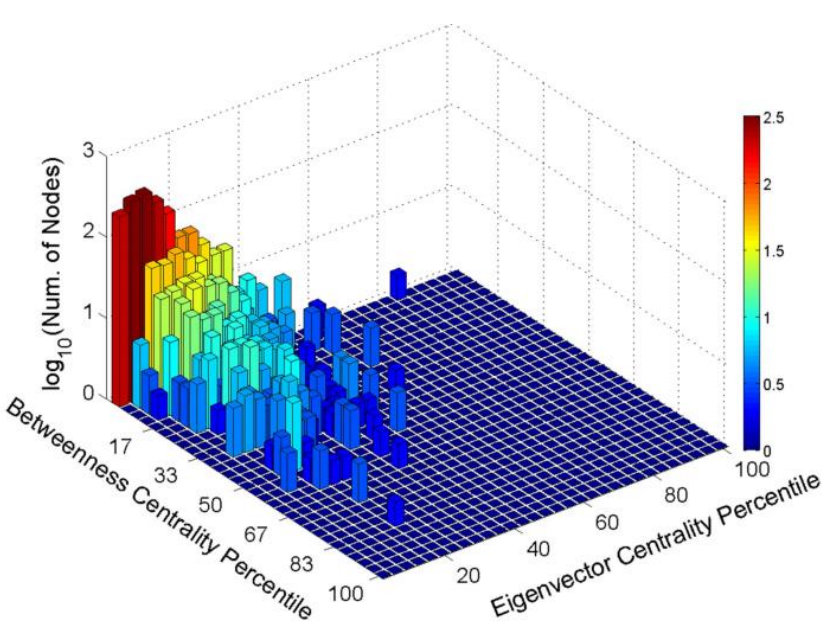

Fig. 1. The number of nodes (logarithmically scaled) corresponding to the intersection of Betweenness Centrality vs. Eigenvector Centrality values. The centrality values are expressed as percentiles to their maximal values.

Fig.1 demonstrates the logarithmically scaled number of nodes corresponding to the $C^{B}$ and $C^{E}$ pairs. The nodes seem to have $C^{E}$ values below $50 \%$ while the $C^{B}$ scale is widely used. This scene outlines that there exists a very small number of nodes having high $C^{E}$ values, while the $C^{B}$ values display a more homogenous decaying characteristic. The trend also shows that highest pairing probability emerges as very small $C^{B}$ 
values ( $<5 \%)$ with a range of $C^{E}$ values between 0 to $30 \%$. The highest $C^{B}$ values seem to pair with $C^{E}$ values around $20 \%$. Also the highest $C^{E}$ values pair with $C^{B}$ values below $5 \%$.

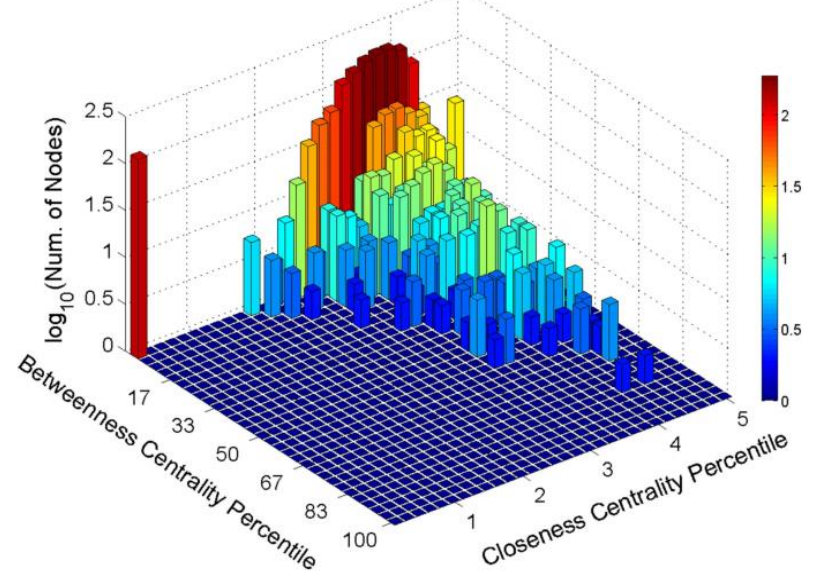

Fig. 2. The number of nodes (logarithmically scaled) corresponding to the intersection of Betweenness Centrality vs. Closeness Centrality values. The centrality values are expressed as percentiles to their maximal values.

Fig.2 shows the logarithmically scaled number of nodes corresponding to the $C^{B}$ and $C^{C}$ pairs. Since the upper $95 \%$ scale of the $C^{C}$ values include very sparse node circumstances, we focused on the first 5\% of the $C^{C}$ values in the graph. Therefore, we can conclude that most of the nodes in the network have small $C^{C}$ values, affected from the spatial dependencies of the network. On the other hand, the nodes display higher $C^{B}$ values compared to $C^{C}$, indicating that the nodes in some way locate in the shortest paths between cities, whether they are not close to the rest of the network. The single distribution of $C^{C}$ cumulates between $2-5 \%$ of the axis, having peak values at $\sim 4 \%$. This is an indicator of the spatial complexity of the network, which leads numerous hops to connect locations in the map, resulting high separation values mentioned in the beginning of the section. As mentioned in Fig.1, $C^{B}$ scale is widely used. The pairing probability between $C^{B}$ and $C^{C}$ values make peak around $4 \%$ of $C^{C}$ and $<5 \%$ of $C^{B}$ values, decaying towards lower closeness and higher betweenness centrality values.

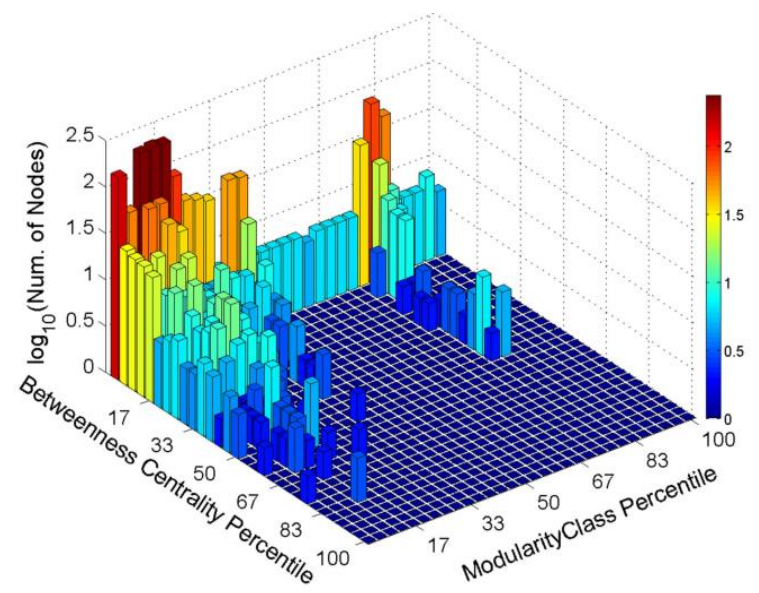

Fig. 3. The number of nodes (logarithmically scaled) corresponding to the intersection of Betweenness Centrality vs. Modularity Class values. The centrality and modularity values are expressed as percentiles to their maximal values.
Fig. 3 demonstrates the pairing characteristics between $C^{B}$ and $M$. We can infer from the figure that both measures display more homogenous distributions compared to the first two plots. The higher $C^{B}$ values emerge for either $M$ values less than $43 \%$, or $M$ values close to $80 \%$. This behavior seems to be mostly driven by the bimodal distribution of Modularity. The most probable pairing occasion is for the lower band $C^{B}$ and $M$ values, while there exist nodes also having low $C^{B}$ together with high modularity values.

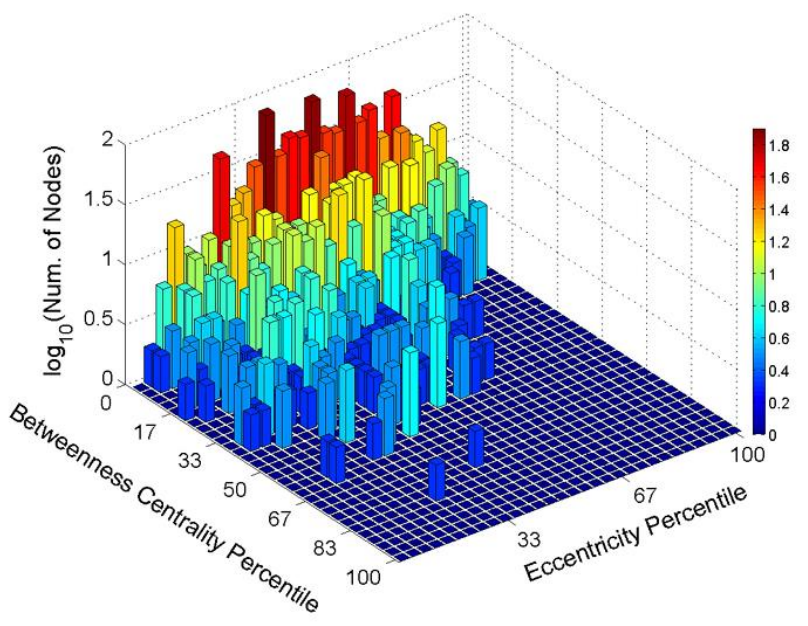

Fig. 4. The number of nodes (logarithmically scaled) corresponding to the intersection of Betweenness Centrality vs. Eccentricity values. The centrality and eccentricity values are expressed as percentiles to their maximal values.

Fig.4 yields the pairing characteristics between $C^{B}$ and $E$. We can infer from the figure that the nodes seem to have $E$ values distributed smoothly through the percentile range while the $C^{B}$ distribution decays with increasing values. The highest pairing probability emerges for small $C^{B}$ values $(<17 \%)$ with the midrange of $E$ values. This property indicates that nodes with high eccentricity values tend to have small betweenness centrality values in the transportation network.

\subsection{Traffic Volume}

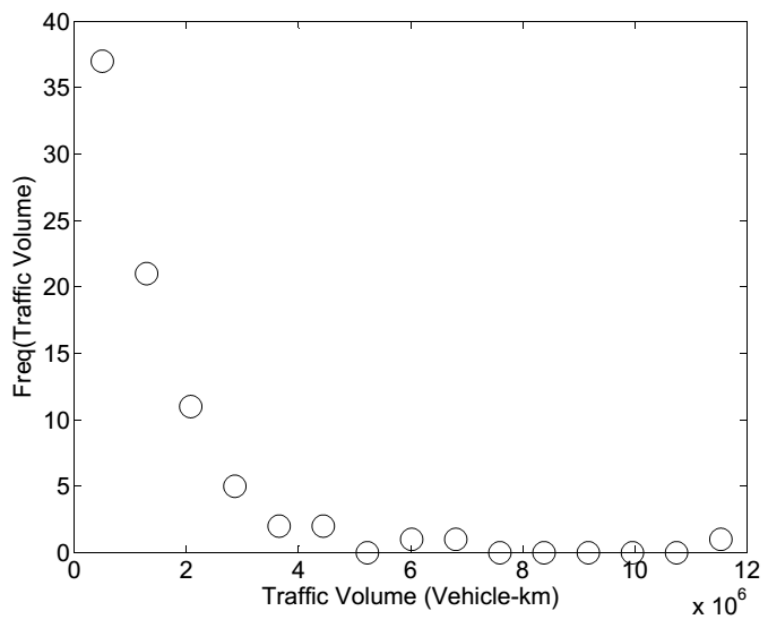

Fig. 5. Histogram for traffic volume in vehicle-km, retrieved from KGM statistics. The volumes are products of vehicle counts with distances covered in kilometers. 
We retrieved the traffic volume data for the cities from the statistics section of KGM [34]. This data set includes traffic volume data with a vehicle-km basis, including the product of the vehicles with the distance covered. We first present the histogram of traffic volume in Fig. 5. This figure indicates a small portion of cities have very high traffic volumes, whereas the probability increases towards small volumes.

To investigate the correlation of traffic volume with network parameters, we present the scattered distribution of five network parameters through the traffic volume in Fig. 6. The data used in these graphs include the traffic volume measures for all 81 cities in Turkey, coupled with the nodes that correspond to the city centers in the network representations. Therefore, it represents only 81 nodes of the network for which a traffic volume data can be retrieved from KGM statistics. The relation between the centrality measures and modularity versus traffic volume do not indicate direct correlations as the graphics display. Only the eccentricity measure implies a correlated trend through the traffic volume range. To quantify the correlations between these five measures and traffic volume, we present the correlation coefficients calculated from the same data as in Table 1.

TABLE I

CORRELATION COEFFICIENTS CALCULATED FOR THE FIVE PARAMETERS VERSUS TRAFFIC VOLUME FOR THE 81 CITY CENTERS

\begin{tabular}{lc}
\hline & CORR. COEFF. \\
\hline CLOSENESS C. & $\mathbf{- 0 , 0 7 6}$ \\
BETWEENNESS C. & $\mathbf{- 0 , 0 3 6}$ \\
EIGENVECTOR C. & $\mathbf{0 , 1 0 2}$ \\
MODULARITY & $\mathbf{0 , 1 5 3}$ \\
ECCENTRICITY & $\mathbf{0 , 3 2 2}$ \\
\hline
\end{tabular}

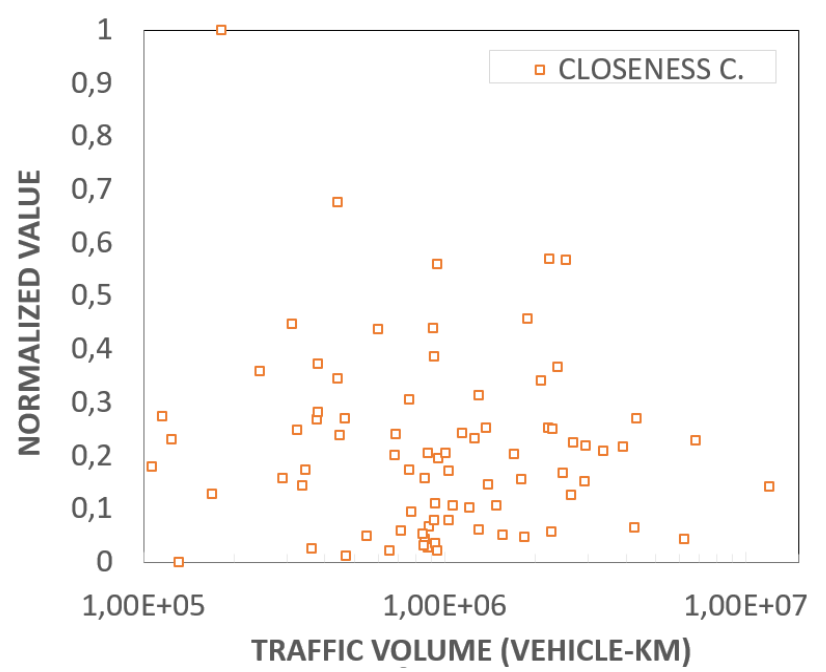

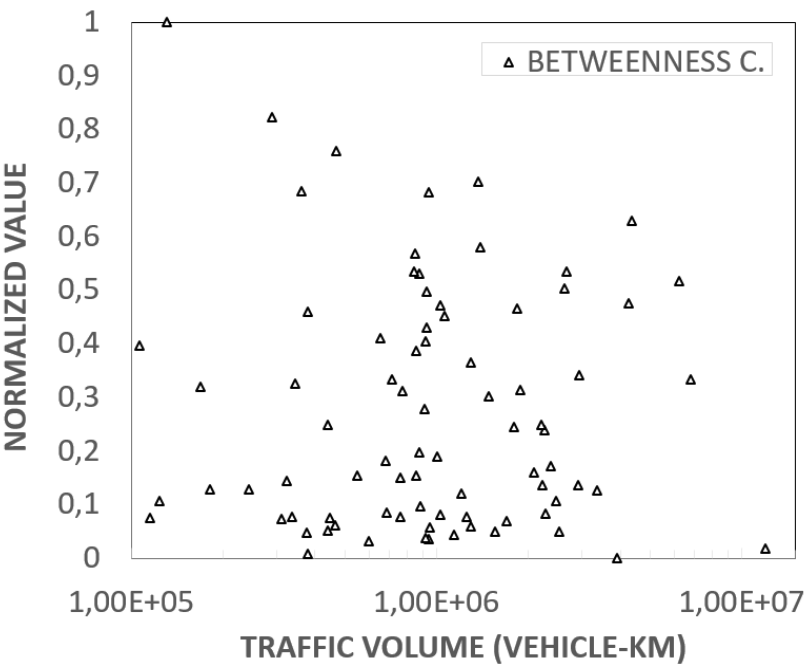
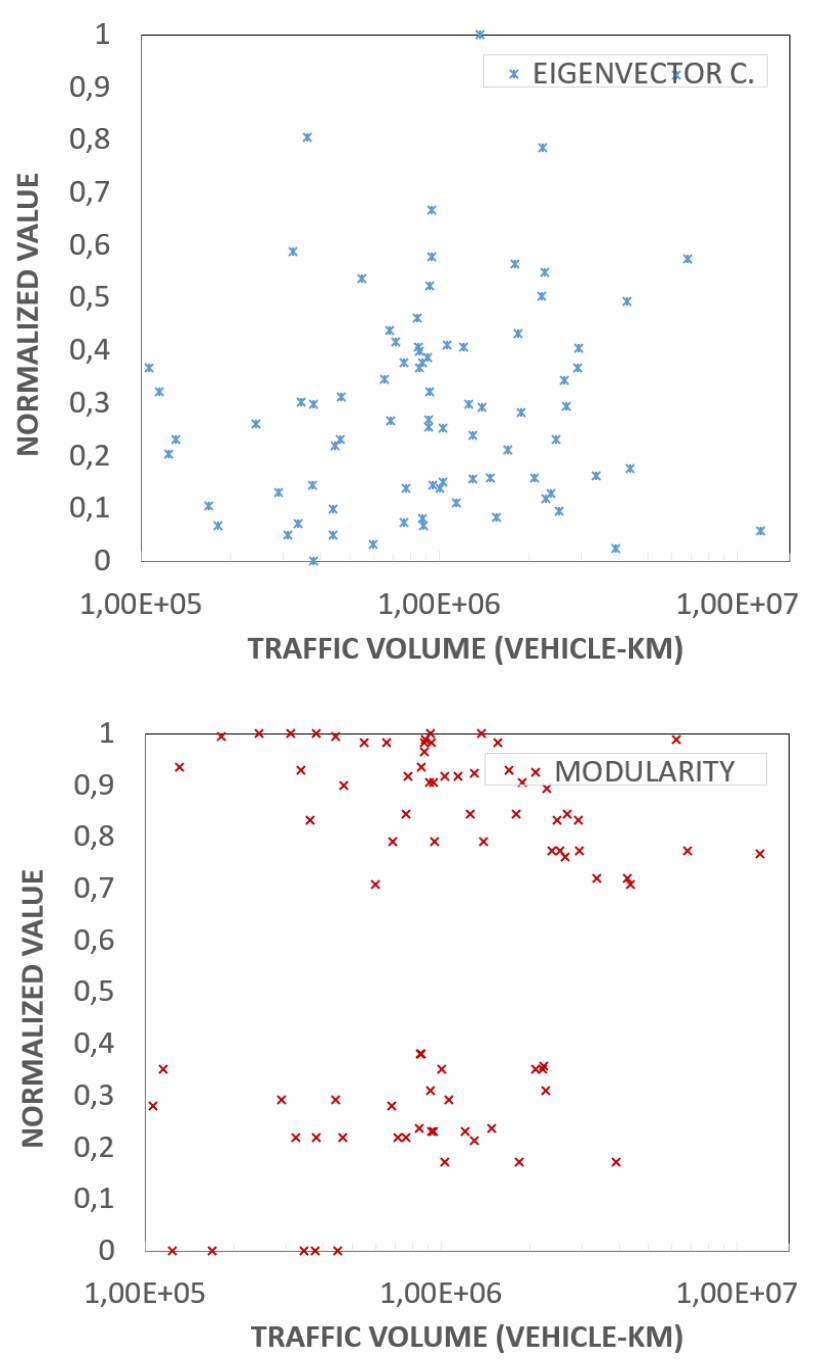


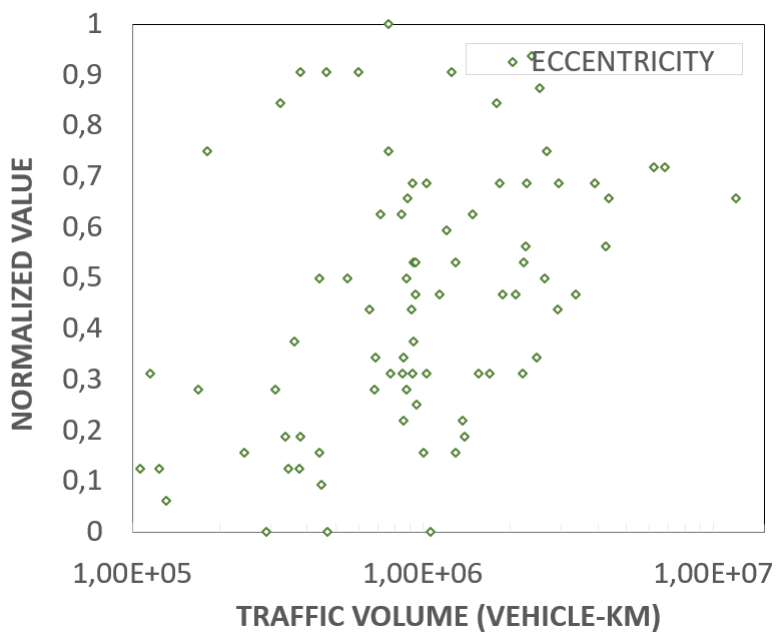

Fig. 6. Scattered distribution of 3 centrality parameters, modularity, and eccentricity through traffic volume in vehicle- $\mathrm{km}$. The parameters in the vertical axis are normalized according to their min-max values. The first panel includes all parameters in one plot, while the other panels display the standalone plots of these parameters vs. traffic volume.

The correlation coefficients presented in Table 1 indicate that the only noteworthy parameter by the means of correlation with the traffic volume is the eccentricity. The main cause for this output may be that higher eccentricity, meaning higher greatest path length with the rest of the cities, results more number of cities which may use the path through that city. In fact, betweenness centrality measure should be expected to imply more correlation with traffic volume, but in this case (a spatially distributed transportation network) shortest paths do not emerge to determine transportation routes. Instead, drivers seem to prefer routes with shortest metric distances or shortest trip durations.

\subsection{Degree Correlations and Assortativity}

Generally, a network is denoted to have degree correlations if the number of links between the nodes with high and lowdegrees is invincibly different from a randomly generated network. In an assortative network, hubs favor connecting to each other and avoid connecting nodes with small degrees. Additionally, small-degree nodes favor connecting to other small-degree nodes. Networks displaying such trends are assortative. Contrary with this definition, in a disassortative network, hubs don't connect to hubs, preferring wiring with small-degree nodes. And the nodes with small degrees tend to connect to hubs, avoiding links to nodes with comparable degrees. If both trends are not observed in a network, i.e. the degree of a node does not affect its neighborhood preferences, such a network is called neutral [35].

To measure this behavior in a network, degree correlation function is derived from the edge matrix of the network. We first measure the average degree of the neighbors of each node as in Eq. 4.

$k_{n n}\left(k_{i}\right)=\frac{1}{k_{i}} \sum_{j=1}^{N} A_{i j} k_{j}$
Then, the degree correlation function is used to calculate the average degree of neighbors for all nodes with degree $\mathrm{k}$ as in Eq. 5.

$k_{n n}(k)=\sum_{k^{\prime}} k^{\prime} P\left(k^{\prime} \mid k\right)$

Where $P\left(k^{\prime} \mid k\right)$ stands for the conditional probability that a node of degree- $k$ will have a neighbor of degree- $k$ '. As a result, $k_{n n}(k)$ denotes the average degree of the neighbors of all nodes with degree $k$ [35]. The $k_{n n}$ vs $k$ plot displays an increasing trend in assortative networks, a decreasing trend in disassortative networks, and a constant trend in neutral networks.

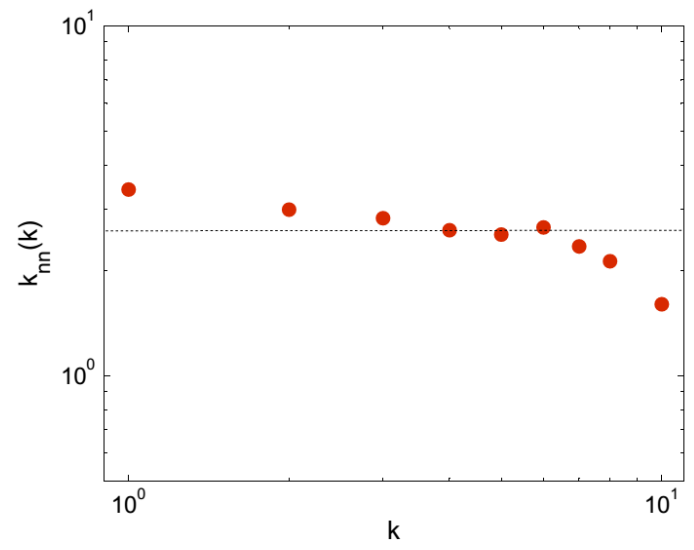

Fig. 7. Degree correlations for the Turkish highway transportation network. The dotted line corresponds to average degree. The decreasing trend of the $k_{n n}(k)$ function indicates that the network is disassortative.

Deriving the $k_{n n}(k)$ plot for our network, we present the degree correlation function in Fig. 7. The decreasing trend is obvious, indicating that the network is disassortative. This behavior is evident in most transportation networks as mentioned in the introduction section.

Another indicator of degree correlations is the degree correlation coefficient, proposed by Newman and calculated with the equation below :

$r=\frac{1}{\sigma_{q}^{2}} \sum_{j k} j k\left(e_{j k}-q_{j} q_{k}\right)$

where

$\sigma_{q}^{2}=\sum_{k} k^{2} q_{k}-\left[\sum_{k} k q_{k}\right]^{2}$

For the detailed extraction of Eq. 6 , the reader is referred to the study of Newman in Ref. [36].

Correlation function is zero in the absence of assortative mixing, while it is positive for assortative or negative for disassortative mixing properties. The real and modeled network examples reported by Newman are also presented in Table 2 [36].

Calculating the assortativity coefficient $r$ for our network, we found $r=-0.1708$, confirming the above inference of disassortativity. We can therefore conclude that the spatially generated urban transportation network studies in this paper yields disassortative mixing property. 
TABLE II

SIZE $n$ AND ASSORTATIVITY COEFFICIENT $r$ FOR A NUMBER OF DIFFERENT NETWORKS, REPORTED BY NEWMAN [36].

\begin{tabular}{|c|c|c|c|}
\hline & network & $n$ & $r$ \\
\hline \multirow{5}{*}{$\begin{array}{l}\frac{n}{4} \\
\overrightarrow{0} \\
3 \\
0 \\
0 \\
0\end{array}$} & physics coauthorship $^{a}$ & 52909 & 0.363 \\
\hline & biology coauthorship $^{a}$ & 1520251 & 0.127 \\
\hline & mathematics coauthorship $^{\mathrm{b}}$ & 253339 & 0.120 \\
\hline & film actor collaborations ${ }^{\mathrm{c}}$ & 449913 & 0.208 \\
\hline & company directors $^{\mathrm{d}}$ & 7673 & 0.276 \\
\hline$\frac{\pi}{\pi}$ & Internet $^{e}$ & 10697 & -0.189 \\
\hline $\bar{\beta}$ & World-Wide Web $^{\mathrm{f}}$ & 269504 & -0.065 \\
\hline$\frac{1}{\sigma}$ & protein interactions ${ }^{\mathrm{g}}$ & 2115 & -0.156 \\
\hline$\stackrel{Ð}{Ð}$ & neural network ${ }^{\mathrm{h}}$ & 307 & -0.163 \\
\hline & food web $^{i}$ & 92 & -0.276 \\
\hline 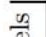 & random graph ${ }^{\mathrm{u}}$ & & 0 \\
\hline 过 & Callaway et al. ${ }^{\mathrm{v}}$ & & $\delta /(1+2 \delta)$ \\
\hline$\stackrel{̊}{g}$ & Barabási and Albert ${ }^{\mathrm{w}}$ & & 0 \\
\hline
\end{tabular}

Depending on Table 2, in which the assortativity coefficients for various networks are reported by Newman, we can figure out that together with the transportation networks mentioned in the introduction section, the disassortative mixing behavior is coherent with some real networks like Internet, World-Wide Web, protein interaction networks, neural networks, food web etc. These types of networks are rather physically connected networks, while the social networks listed as the first group of Table 2 display assortative mixing property.

\section{CONCLUSIONS AND FUTURE WORK}

Studying the centrality, modularity and assortativity properties of the Turkish highway transportation network, composed of physical connections (roads) between the junctions of roads as edges, we presented 3D comparative plots of the centrality and modularity measures first. We outlined that the betweenness centrality scale used in all the three $3 \mathrm{D}$ percentile plots has a homogenous distribution, meaning that the network includes nodes of various $C^{B}$ values with a decreasing trend. Nodes have eigenvector centrality measures stuck in the lower $50 \%$ band of the $C^{E}$ scale, while the highest $C^{B}$ values seem to pair with $C^{E}$ values around $20 \%$. The nodes with highest $C^{E}$ values tend to have $C^{B}$ values below $5 \%$.

Focusing on the closeness centrality measure, we see that almost all the nodes are of $5 \%$ closeness to the rest of the network. This is an expected result originating from the spatial dependencies of the network. Having relatively small closeness values, the nodes display higher betweenness centrality values, indicating that the nodes in some way locate in the shortest paths between cities whether they are not close to the rest of the network. The single distribution of $C^{C}$ ranging between $2-5 \%$ of the axis indicates that the network has a spatially complex structure, resulting high separation between nodes. $C^{B}$ and $C^{C}$ values mostly pair around $4 \%$ of $C^{C}$ and $<5 \%$ of $C^{B}$ scales, decaying towards lower closeness and higher betweenness centrality values.

The modularity measure, conveying information about the tendency of nodes to form modules, is recently reported to have very high values for this network [26], therefore labels the network as highly modular. The single distribution of Modularity displays bimodal character while it pairs with higher betweenness centrality values around $43 \%$ and $80 \%$ of the scale. The pairing probability peaks for lower betweenness and modularity.

The eccentricity measure, quantifying the maximal shortest distance of a node to the rest of the nodes is nearly uniformly distributed over betweenness centrality, whereas it is the most correlated measure with the traffic volume of the cities. We conclude that the uncorrelated behavior of traffic volume with the centrality measures is mostly driven by the selection of trip routes by drivers regarding with the main roads/highways that make the travel shorter in metric distance or time. These routes generally may not be the shortest paths of the graph.

The concept of assortativity in a network probes the emergence of degree correlations of the paired nodes. The quantitative way of testing this phenomenon is the assortativity coefficient $r$ proposed by Newman, which we found $r=-0.1708$ for our network. Together with the $\mathrm{k}_{\mathrm{nn}}(\mathrm{k})$ function which yields negative slope for our network, both indicators label our network as disassortative, coherent with the real networks with "more physical" wiring mechanisms.

As a future work, this study can be extended to include suburban roads which are not covered in this study. Also, more detailed transportation networks can be constructed via image processing techniques applied over satellite images.

\section{ACKNOWLEDGMENT}

The author thanks Sabah Bashir Salem Rashed for sharing raw data supplied from KGM maps.

\section{REFERENCES}

[1] H. Jeong, S. P. Mason, A.-L. Barabási, and Z. N. Oltvai, "Lethality and centrality in protein networks," arXiv preprint cond-mat/0105306, 2001.

[2] D. A. Fell and A. Wagner, "The small world of metabolism," Nature biotechnology, vol. 18, p. 1121, 2000.

[3] I. Hartmann-Sonntag, A. Scharnhorst, and W. Ebeling, "Modelling selforganization and innovation processes in networks," arXiv preprint cond-mat/0406425, 2004

[4] J. M. Montoya, S. L. Pimm, and R. V. Solé, "Ecological networks and their fragility," Nature, vol. 442, p. 259, 2006.

[5] M. E. J. Newman, "The structure of scientific collaboration networks," Proceedings of the National Academy of Sciences of the United States of America, vol. 98, pp. 404-409, Jan 2001.

[6] A. Cavusoglu and I. Turker, "Scientific collaboration network of Turkey," Chaos Solitons \& Fractals, vol. 57, pp. 9-18, Dec 2013.

[7] A. Ferligoj, L. Kronegger, F. Mali, T. A. B. Snijders, and P. Doreian, "Scientific collaboration dynamics in a national scientific system," Scientometrics, vol. 104, pp. 985-1012, Sep 2015.

[8] A. M. Manago, T. Taylor, and P. M. Greenfield, "Me and my 400 friends: the anatomy of college students' Facebook networks, their communication patterns, and well-being," Developmental psychology, vol. 48, p. 369, 2012.

[9] A. L. Barabási, R. Albert, and H. Jeong, "Scale-free characteristics of random networks: the topology of the World-Wide Web," Physica A, vol. 281, pp. 69-77, Jun 2000.

[10] A. E. Motter, S. A. Myers, M. Anghel, and T. Nishikawa, "Spontaneous synchrony in power-grid networks," arXiv preprint arXiv:1302.1914, 2013.

[11] G. A. Pagani and M. Aiello, "The power grid as a complex network: a survey," Physica A: Statistical Mechanics and its Applications, vol. 392, pp. 2688-2700, 2013.

[12] M. E. Newman, "The structure and function of networks," Computer Physics Communications, vol. 147, pp. 40-45, 2002.

[13] M. G. Bell and Y. Iida, "Transportation network analysis," 1997.

[14] R. Guimera, S. Mossa, A. Turtschi, and L. N. Amaral, "The worldwide air transportation network: Anomalous centrality, community structure, and 
cities' global roles," Proceedings of the National Academy of Sciences, vol. 102, pp. 7794-7799, 2005.

[15] G. Bagler, "Analysis of the airport network of India as a complex weighted network," Physica A: Statistical Mechanics and its Applications, vol. 387, pp. 2972-2980, 2008.

[16] Z. Xu and R. Harriss, "Exploring the structure of the US intercity passenger air transportation network: a weighted complex network approach," GeoJournal, vol. 73, p. 87, 2008.

[17] H. Soh, S. Lim, T. Y. Zhang, X. J. Fu, G. K. K. Lee, T. G. G. Hung, et al., "Weighted complex network analysis of travel routes on the Singapore public transportation system," Physica a-Statistical Mechanics and Its Applications, vol. 389, pp. 5852-5863, Dec 2010.

[18] Y. Li, W. Zhou, and S.-j. Guo, "An Analysis of Complexity of Public Transportation Network in Shanghai [J]," Systems Engineering, vol. 1, p. 006, 2007.

[19] D. Tsiotas and S. Polyzos, "Introducing a new centrality measure from the transportation network analysis in Greece," Annals of Operations Research, vol. 227, pp. 93-117, 2015.

[20] V. Latora and M. Marchiori, "Is the Boston subway a small-world network?," Physica A: Statistical Mechanics and its Applications, vol. 314, pp. 109-113, 2002.

[21] H.-Y. Shih, "Network characteristics of drive tourism destinations: An application of network analysis in tourism," Tourism Management, vol. 27, pp. 1029-1039, 2006.

[22] D. M. Scott, D. C. Novak, L. Aultman-Hall, and F. Guo, "Network robustness index: A new method for identifying critical links and evaluating the performance of transportation networks," Journal of Transport Geography, vol. 14, pp. 215-227, 2006.

[23] A. Chen, H. Yang, H. K. Lo, and W. H. Tang, "Capacity reliability of a road network: an assessment methodology and numerical results," Transportation Research Part B: Methodological, vol. 36, pp. 225-252, 2002.

[24] S. Porta, P. Crucitti, and V. Latora, "The network analysis of urban streets: a primal approach," Environment and Planning B: planning and design, vol. 33, pp. 705-725, 2006.

[25] S. Porta, P. Crucitti, and V. Latora, "The network analysis of urban streets: a dual approach," Physica A: Statistical Mechanics and its Applications, vol. 369, pp. 853-866, 2006.

[26] İ. Türker, S. O. Tan, and S. B. S. Rashed, "Extracting the connectivity properties of the Turkish highway transportation network," presented at the ICRES, Kuşadası, Aydın, 2017.

[27] (2017). Karayolları Genel Müdürlüğü Bilgi İşlem Dairesi, "Haritalar". Available: http://www.kgm.gov.tr/Sayfalar/KGM/SiteTr/Root/Haritalar.aspx

[28] P. Crucitti, V. Latora, and S. Porta, "Centrality measures in spatial networks of urban streets," Physical Review E, vol. 73, p. 036125, 2006.

[29] S. Wasserman and K. Faust, Social network analysis: Methods and applications vol. 8: Cambridge university press, 1994.

[30] P. Bonacich, "Some unique properties of eigenvector centrality," Social networks, vol. 29, pp. 555-564, 2007.

[31] P. Dankelmann, W. Goddard, and C. S. Swart, "The average eccentricity of a graph and its subgraphs," Utilitas Mathematica, vol. 65, pp. 41-52, 2004.

[32] M. E. J. Newman, "Modularity and community structure in networks," Proceedings of the National Academy of Sciences, vol. 103, pp. 8577$8582,2006$.

[33] M. Bastian, S. Heymann, and M. Jacomy, "Gephi: an open source software for exploring and manipulating networks," presented at the International AAAI Conference on Weblogs and Social Media, San Jose, California, 2009.

[34] "Karayolları Genel Müdürlüğü Bilgi İşlem Dairesi, "Trafik ve Ulaşım Bilgileri"," 2017.

[35] A. L. Barabási, Network Science. Cambridge: Cambridge University Press, 2016.

[36] M. E. Newman, "Assortative mixing in networks," Physical review letters, vol. 89, p. 208701, 2002.

\section{BIOGRAPHIES}

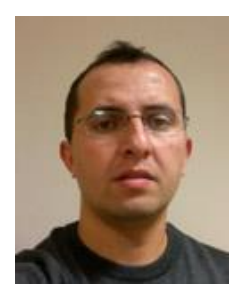

İlker TÜRKER was born in Karabuk, Turkey, in 1980. He received his B.S. Degree from Istanbul Technical University, Dept. of Electronics and Telecommunications, in 2001, M.S. degree from Zonguldak Karaelmas University, Dept. of Electrics and Electronics, in 2006 and Ph.D. degree in computer software, from Karabuk University, Dept. of Computer Engineering, in 2013. He has been an Assistant Professor with the Computer Engineering department, Karabuk University. He studies on data mining, complex network analysis and modeling, neurocomputing. He is also Assistant Editor-in-Chief in JESTECH, an international ESCI-indexed journal about engineering, science and technology, which is published by Elsevier BV. 